\title{
SMILE ANALYSIS AND ESTHETIC PERCEPTION OF A SAMPLE OF SAUDI POPULATION
}

\author{
Kholood Alhussami* and Hani M. Nassar**
}

\begin{abstract}
Aim: The aim of this observational study was to assess the different esthetic smile criteria of adults in Saudi Arabia. Further goals were to find the predominant dental shades and assess subjects' esthetic perception in a sample of Saudi community.

Materials and Methods: Thirty subjects were recruited from King Abdulaziz University Dental Hospital in Jeddah, Saudi Arabia. Subjects were between 18-50 years of age with intact upper anterior teeth and healthy gingiva. All participants were photographed and the following smile parameters were recorded: tooth geometry, shade, midlines, smile arc, and upper lip position. Parameters were plugged in a dedicated smile analysis software and the golden proportion values were obtained. Data were analyzed using Chi Square and Student's t-tests according to variables tested at 0.05 significance level.

Results: Regardless of age and sex, $70 \%$ of the sample has an average lip position, and consonant smile arc was the prevalent form among $73.3 \%$ of the sample. The most common shade was A2 regardless of tooth type or position. Almost half of the participants were satisfied with their overall appearance and only 6 subjects were not satisfied with the size of their teeth. Overall, the golden proportion was not present in the tested sample.

Conclusion: Despite the absence of golden proportion, the majority of the sample tested was satisfied with their smile parameters. The prevalent shade was A2 in both genders.
\end{abstract}

KEYWORDS: Smile analysis; Esthetic perception; shade; and golden proportion

\section{INTRODUCTION}

A brilliant smile is an important feature in the cosmetic world. It is a sign of the beauty that gives a nice presentation of a person and a good impact on perception and self-motivation (Kaur et al., 2011). Further, a good smile is considered as one of the first lines of non-verbal communication that makes

Intern, Faculty of Dentistry, King Abdulaziz University, Jeddah, Saudi Arabia.

** Assistant professor, Department of Restorative Dentistry, Faculty of Dentistry, King Abdulaziz University, Jeddah, Saudi Arabia. 
it more important in audience-faced jobs such as actors and TV shows hosts (Singla \& Lehl, 2014). Nowadays, the awareness of the population about their dental esthetic problems that affect their smiles is increased with the spread of new techniques' and materials' advertisement; especially for those who received dental education (Al-Marzok et al., 2013; Al Shahrani, 2014).

An ideal smile has several parameters that should be considered while restoring teeth. (Al-johany \& Alzahrani, 2011). These components are smile arc, facial upper and lower midlines, lip position, upper lip curvature, and structural parallelism among others. The correlation between these parameters can be altered to achieve different smiles and appearance. For example, the relation between the upper lip and the gum appearance could totally transform the appearance from a gummy smile to a nice and more natural smile with a convenient amount of a gum exposure (Sudhakar \& Vishwanath, 2014 )diagnose and resolve esthetic problems predictably. Our ultimate goal is to achieve pleasing composition in the smile by creating an arrangement of various esthetic elements. One of the most important tasks in esthetic dentistry is creating harmonious proportions between the widths of maxillary anterior teeth when restoring or replacing these teeth This review article describes application of the Golden Proportion and Red Proportion in dentistry also the smile design for completely edentulous and for full mouth rehabilitation. Also the future scope for designing smile. (Sudhakar \& Vishwanath, 2014). Any alteration in the size of an upper anterior tooth by a bad esthetic restoration could produce an unpleasing smile and lower the esthetic perception especially in females (Al-Marzok et al., 2013; Al Shahrani, 2014; Tin-Oo et al., 2011).

Another parameter that is receiving a lot of attention is the golden proportion which is an important esthetic guide. Even though this formula can be used to achieve an esthetic outcome, it is not essential to follow it and some well-known celebrity's smiles do not follow the golden proportion, yet their smiles are considered pleasing (Al-johany \& Alzahrani, 2011). Shade selection is a daily perplexing procedure that faces every dentist to get the high level of patient satisfaction after the treatment. Methods are different but the goal is one, which is a productive natural appearance. Other records should be considered to finalize the restoration such as surface texture on light reflection (Mehta et al., 2014). The present study aimed to evaluate the presence of different esthetic smile criteria of adults in Jeddah. A secondary objective was to evaluate the smile perception of this sample.

\section{MATERIALS AND METHODS}

Thirty adult patients (15 male and 15 female) aging between 18 and 50 years were included in the study. The study was approved by the ethical committee of King Abdulaziz University Dental Hospital (Jeddah, Saudi Arabia) which is the facility from which the subjects were recruited and where the study was undergone.

In addition to age, other inclusion criteria for enrollment were: presence of sound upper permanent anterior teeth and first premolars, absence of any large restorations or prosthetic crowns in the upper anterior teeth, absence of attrition or abrasion in the upper anterior teeth, and absence of periodontal conditions. Patients with history of orthodontic treatment, previous major trauma, plastic surgery in the face, or history of recent bleaching or endodontic treatment in upper anterior teeth and first premolars were excluded.

Participants signed a consent form and filled up a questionnaire that included questions about selfperception of their smiles. After filling the survey, two frontal photos and three intraoral photographs (frontal, upper and lower occlusal) were taken from each participant with a digital camera (Nikon Coolpix P100, Nikon Inc., Tokyo, Japan). In order to standardize the process, all photographs were shot with fixed space between the camera and the patient's face while the feet were on the floor and the head touching a white wall and the interpupillary 
line was parallel to the floor. The patient looked at a reference point during taking the photo.

A detailed clinical examination was carried out to record the components of each patient's smile. The shade of the anterior and premolar teeth were recorded by two evaluators and two methods: Vitapan composite shade guide (classic A1-D4; Vita North America, Yorba Linda, California, United States) and tooth color comparator digital shade matching device (Linker Dental Group Limited, Henan, China). The shade was selected from the center of the labial surface of the tooth and under daylight. Tooth measurements (height and widths in millimeters) were measured by a digital caliper.

Relation of the upper lip to the amount of gingival appearance were recorded into one of three categories: a high smile revealing the entire cervico-incisal length of the maxillary anterior teeth with an adjoining band of gingiva, an average smile reveals $75 \%$ to $100 \%$ of the maxillary height of upper anterior teeth and the interproximal gingiva only, and a low smile line displaying $75 \%$ of the upper anterior teeth or less. Buccal corridors define as black triangles in the corner of the mouth during smiling were recorded as average, wide, or absent. In addition, the number of teeth displayed during smiling was also recorded while taking into consideration which teeth are touching the lower lip. Upper and lower midlines were also recorded.

Smile arc was evaluated in photographs by drawing a line connecting the incisal edges of the upper anterior teeth and correlating it to the general outline of the lower lip. Smile arcs were recorded as consonant, flat, increased, or reversed). Lastly, the golden proportion (Fig. 1) was evaluated by Smile Designer Pro software (Tasty Tech Ltd., Toronto, Canada) after inputting all the above-mentioned parameters.

Chi Square and Student's t-tests were used to analyze the data according to the type of variable tested (SPSS ver. 20, SPSS Inc., Chicago, Illinois, USA) at 0.05 significance level.

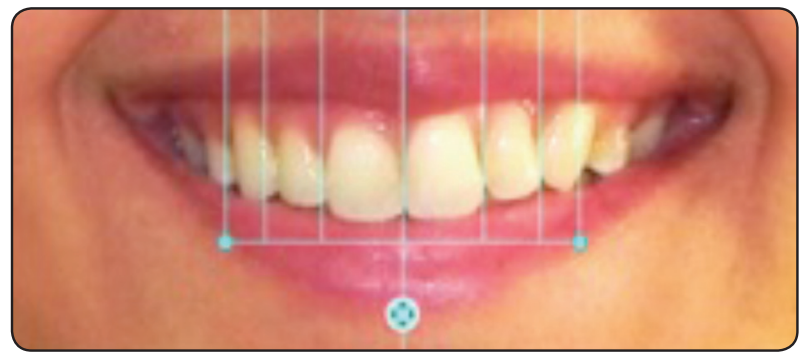

Fig. (1) A design tool provided by Smile Designer Pro overlaid the subject's photograph.

\section{RESULTS}

There was a trend for female subjects having smaller teeth overall; however, the difference between measurements was not statistically different between genders (Table 1). The golden proportion was not existent in the study population. Regardless of age and gender, $70 \%$ of the sample has average lip position that shows the entire cervico-incisal length of maxillary anterior teeth along with the interproximal gingiva. While $20 \%$ have high lip line with complete display of the cervico-gingival length and contiguous band of gingival tissues. Low lip position was presented in $10 \%$ only (Fig. 2).

The cumulative agreement between conventional shade matching method and digital shade matching device was $17 \%$ indicating poor agreement between the two techniques. However, digital shade matching consistently recorded lighter shades compared to the conventional method. The most frequent shade of teeth of the sample was A2 (43.3\%) followed by A3 $(21.6 \%)$ and the least percentage was for B1 and B3 $(0.41 \%)$. There was no statistically significant difference between genders in regards to shades.

Consonant smile arc was the most prevalent form and was present among $73.3 \%$ of the sample that is considered when the two lines were parallel to each other. Flat smile line was found in $20 \%$ of the sample; while reversed or reduced arc was present in only $7 \%$ of the sample. More than $50 \%$ of the participants gave a smile extended to the second premolar followed by first premolars. 
TABLE (1) Mean and standard deviation (in parenthesis) of measurements of upper anterior teeth and premolars for the entire study population.

\begin{tabular}{|c|c|c|}
\hline Tooth & Mean Height (mm) & Mean Width (mm) \\
\hline 11 & $10.3(0.96)$ & $8.8(0.8)$ \\
12 & $8.37(0.95)$ & $6.7(0.6)$ \\
13 & $9.42(1.17)$ & $8.23(0.96)$ \\
14 & $9.78(1.3)$ & $7.05(0.76)$ \\
21 & $10.2(1.06)$ & $8.93(0.81)$ \\
22 & $8.68(1.04)$ & $7.01(0.94)$ \\
23 & $7.37(1.28)$ & $8.21(1.1)$ \\
24 & $7.68(1.06)$ & $7.17(0.76)$ \\
\hline
\end{tabular}

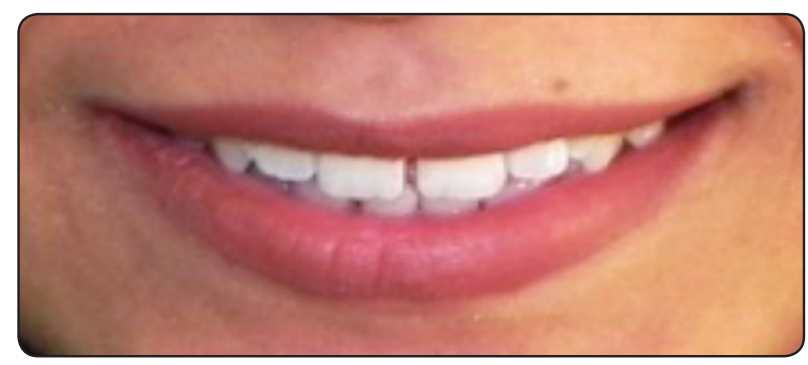

Fig. (2) Low lip position with less than $75 \%$ of the entire length of the maxillary anterior teeth.

The majority of participants had centered upper midline $(90 \%)$ in relation to the facial midline and 3 subjects presented with shifted midline to the right. The lower midline was found mostly shifted whether to the right or left and centered in 9 subjects only. The most prevalent arch form was oval which was found in $76.6 \%$ and $60.0 \%$ of upper and lower arches, respectively. The triangular form was the least prevalent (10\% of both arches).

Average sized buccal corridors was found in $40 \%$ in the right side while $53.3 \%$ in the left side. Only two subjects were presented with no left buccal corridor and 9 subjects for the right side (Fig.3). Wide corridors were displayed symmetrically or combined with another size.

For self-perception of the smile, sixty percent of the subjects were highly satisfied with their overall appearance. Once they were asked about their teeth color, females showed less satisfaction while males were fairly satisfied (Fig.4); even though it was found that both genders desired their teeth to be whiter (90\%). Only one male was not satisfied about his teeth size while the majority of the sample was highly satisfied (Fig.5).

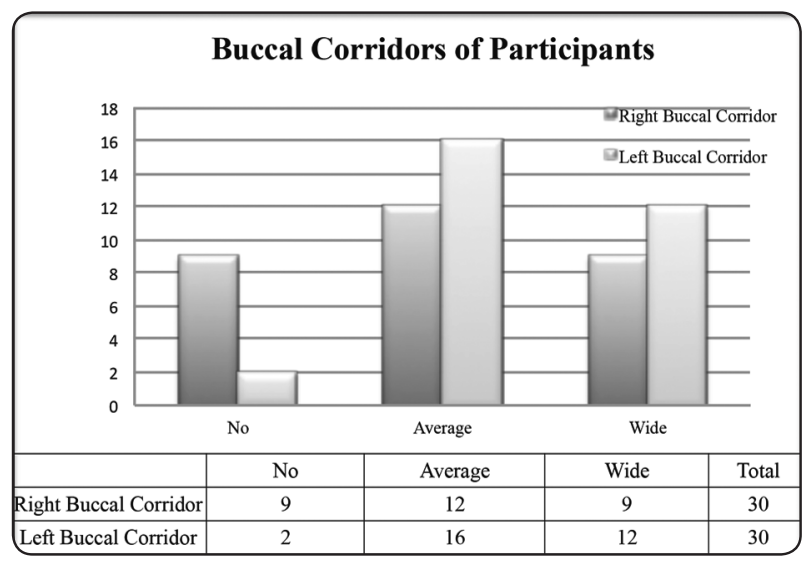

Fig. (3) Proportions of right and left buccal corridors of subjects from the left and right sides.

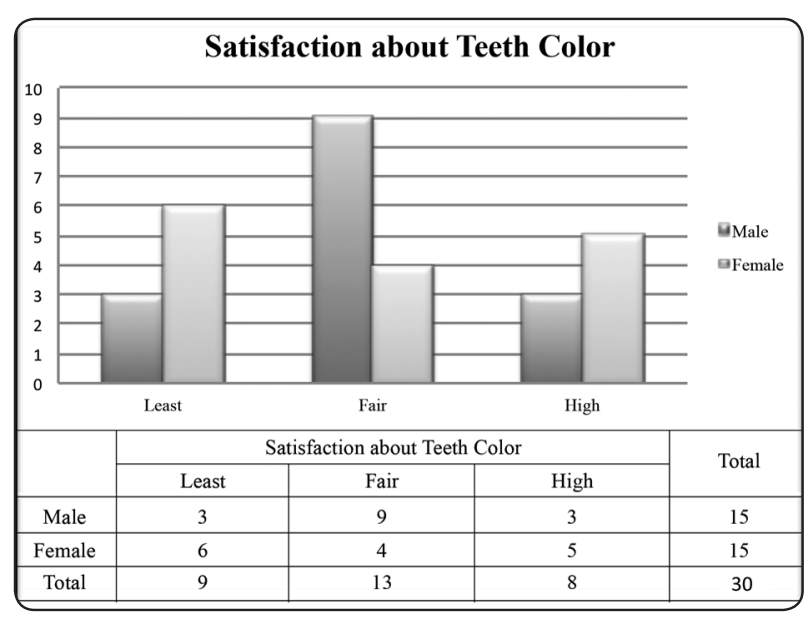

Fig. (4) The last row in the table is not showing properly. 


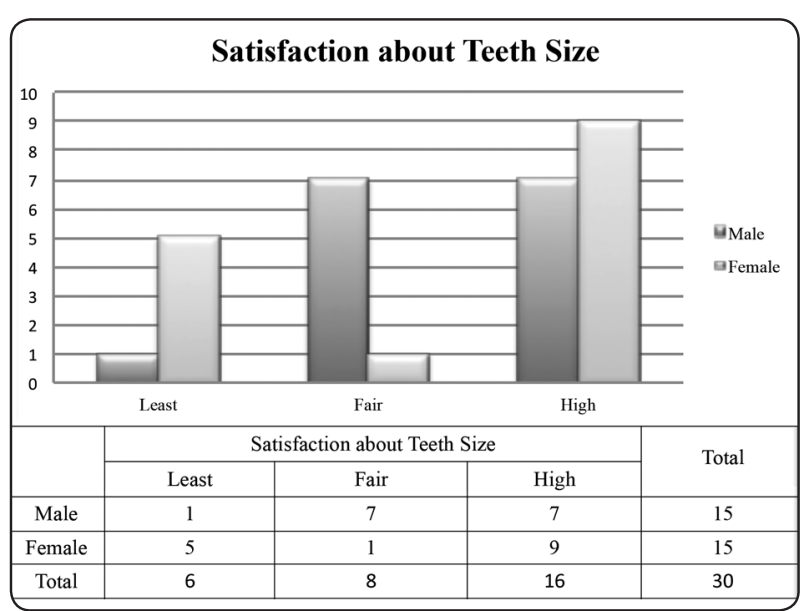

Fig. (5) Proportions of sample's satisfaction about their teeth size.

\section{DISCUSSION}

Achieving a harmonious and attractive smile is paramount in Operative and Esthetic dentistry. However, in order to achieve this, the dentist must be familiar with the characteristics of a pleasant smile and the prevalence of each parameter. In addition, a general knowledge of how patients perceive their smiles is also important. Thus, the main objectives of this study were to evaluate the esthetic smile parameters and to assess subjects' esthetic perception in a sample of Saudi population attending a local government university dental hospital.

As previously stated, there are many factors affect the smile design. Golden ratio/ proportion is considered as one of the main guidelines to create harmonious proportions between the widths of anterior maxillary teeth (Mahshid et al., 2004). In this study, a frontal photograph was taken for each subject while smiling and Smile Designer Pro software was used to evaluate the existence of the golden ratio in order to facilitate the process and save time. In the present sample, there was no existence of golden proportion with the variety of natural teeth proportions tested. Still, the golden proportion is a reported finding in different societies other than Saudi population, such as the Malaysian population (Al-Marzok et al., 2013; Mahshid et al.,
2004). Subject's gender was not a significant factor in this study as seen in Indian population, but ethnic differences could have a role in large countries due to cultural diversity (Chander et al., 2012). This will require more investigation in different regions.

The existence of the variation in hue, value and, chroma of teeth, which gives the three dimensional depth and characteristics, increases the difficulty of shade matching (Mehta et al., 2014). There are two methods of shade selection in dentistry: visual and instrumental with multiple factors affecting the process such as translucency, contour, and surface texture (Mehta et al., 2014). In this study, Vitapan Classical shade guide was used and compared to a digital method (Tooth Color Comparator). The tooth body was selected to be the solely part for shade matching in all the maxillary anterior teeth and first premolars. With the limitation of the classical shade guide, A2 shade was the most prevalent shade in the sample followed by A3 shade, regardless the tooth position and the gender. This finding was confirmed in a previous study carried out by Rodrigues et al., using the new version of the conventional shade guide, which is $3 \mathrm{D}$ master shade guide, in respect to the age group (Rodrigues et al., 2012). This result indicates that teeth color changes as age advances as seen in Young et al.'s study (1994). Tooth Color Comparator was used in this study to compare the shade matching with the conventional method used in the school. The agreement as shown in the results is poor (17\%) as the digital devise gave lighter shades. Thus, it is not advisable to rely solely on the digital device for shade matching. In contrast, Paul et al. (2002) found that spectrophotometric devices are more accurate and more reproducible compared with conventional shade guides. Bahannan (2014) at \u03b1 $=0.05$ and with $\mathrm{P}<0.05$ indicating significance. Results Among the participants, $36.3 \%$ visually selected the correct shade, and $80.4 \%$ did so using the Easy Shade Compact machine. Experience $(\mathrm{P}=0.177$ also confirmed that the spectrophotometric devices were significantly better than the conventional visual method, regardless of the experience. 
Passing to another esthetic factor, which is the upper lip position. In this study, it was found that the majority of the sample, which presented by $70 \%$, has an average lip position with optimum gingival display as seen in Indian females as well (Goel \& Patnaik, 2013; Kaur et al., 2011). Al-Johany et al. (2011) reported that $80 \%$ of the chosen photographs of female celebrities, which were voted by lay people to have beautiful smiles, presented with average upper lip position. Another study on Caucasian female subjects showed the highest percentage $(73.7 \%)$ for average position too, which makes it the most prevalent position (Tjan, Miller, \& The, 1984). Consonant smile arc is considered when the incisal edges of the maxillary anterior teeth are parallel to the upper border of the lower lip. In the present study, it was found that the most prevalent smile arc category in subjects (73\%) regardless of age and gender. This is in a agreement with Al-Johany et al. (2011) who recorded a high percentage of such design (78\%) among female celebrities in their study.

The number of teeth showing while smiling is another component of an esthetic smile. In the present study, second premolars were the last teeth showed in the majority of tested subjects which is similar to the Caucasian society (Nold et al., 2014)52 men and Panjabi females (Kaur et al., 2011). Dong et al. (1999) reported that an attractive smile had the overall cervicoincisal length of the maxillary anterior teeth shown between the upper and lower lips, an upward curved or straight smile with a parallel smile arc to the lower lip, and displayed up to the first molar.

Deviation in facial or dental midlines could affect the beauty and attractiveness of the face. In this study, upper midline was checked on site in relation with the facial midline while lower midline was observed from the photographs of patients' retracted smiles. It was found that the preponderance of the sample has centered upper midline to the facial midline (90\%), which is in agreement with a study by Nold et al.
(2014)52 men in Caucasians. Lower midline was often shifted either to right or to the left in relation to the upper midline which mostly seen associated with crowded lower anterior teeth. The sample tends to have oval dental arches as confirmed in the latter study (Nold et al., 2014)52 men. Regardless of gender, smile attractiveness increases when the buccal corridors are narrow or absent (Moore et al., 2005; Oshagh et al., 2010).

Satisfaction about different esthetic parameters differs from one person to another according to the level of awareness (Al Shahrani, 2014). Females tend to be more unsatisfied about their teeth shape and color more than male subjects (Al Shahrani, 2014; Tin-Oo et al., 2011). However, in the present study, the majority of the sample was fairly to highly satisfied with their teeth shape. Female subjects expressed less satisfaction about their teeth color while males were mostly neutral about the color. Ninety percent of the subjects desired their teeth to be whiter as recorded in a Brazilian sample (Silva et al., 2012). There was a high percentage of high satisfaction similarly in male and female about overall appearance. The awareness and dental education affect the self-perception inversely (Al Shahrani, 2014) which is similar to our findings (data not shown).

Within the limitations of the study, we can conclude that the golden proportion was nonexistent in the entire sample which goes with other studies indicating that it is not required in order to have patients feel satisfied with their smiles. However, this satisfaction is affected by gender. The most prevalent smile parameters present were light shades, small buccal corridors and consonant smile arcs.

\section{ACKNOWLEDGEMENTS}

The authors would like to express their sincere thanks to Dr. Ahmed Shukri for his assistance with the study. 


\section{REFERENCES}

1. Kaur G, Kaushal S, Patnaik V.V. G, 2011. The Anatomy of A Smile. J Med Coll Chandigrah. 1(1):20-3.

2. Singla S, Lehl G, 2014. Smile analysis in orthodontics. Indian J Oral Sci. 5(2):49.

3. Al-Marzok MI, Majeed KRA, Ibrahim IK, 2013. Evaluation of maxillary anterior teeth and their relation to the golden proportion in malaysian population. BMC Oral Health. 13(1):9.

4. Al Shahrani I, 2014. Self-perception of personal dental appearance among students of King Khaled University Abha, Saudi Arabia. Eur J Gen Dent. 3(3):181.

5. Al-johany SS,Alzahrani AH, 2011. Evaluation of Different Esthetic Smile Criteria. Int J Prosthodont. 15-7.

6. Sudhakar DN, Vishwanath DA, 2014. Smile Esthetics - A Literature Review. IOSR J Dent Med Sci. 13(1):32-6.

7. Tin-Oo MM, Saddki N, Hassan N, 2011. Factors influencing patient satisfaction with dental appearance and treatments they desire to improve aesthetics. BMC Oral Health. 11(1):6.

8. Mehta R, Pande S, Nanda S, Nanda T, 2014. Shade Selection: Blending of Conventional and Digital Methods - An Updated Review. Journal of Oral Health \& Community. 1(4):5-9.

9. Mahshid M, Khoshvaghti A, Varshosaz M, Vallaei N, 2004. Evaluation of "Golden Proportion" in Individuals with an Esthetic Smile. J Esthet Restor Dent. 16(3):185-193.

10. Chander NG, Kumar VV, Rangarajan V, 2012. Golden proportion assessment between maxillary and mandibular teeth on Indian population. J Adv Prosthodont. 4(2):72-5.

11. Rodrigues S, Shetty SR, Prithviraj DR, 2012. An evaluation of shade differences between natural anterior teeth in different age groups and gender using commercially available shade guides. J Indian Prosthodont Soc.; 12(4):222-30.

12. Young L, Glaros AG, Moore DJ, Collins JF,1994. Assessing shade differences in acrylic resin denture and natural teeth. J Prosthet Dent. 71(6):575-80.

13. Paul S, Peter a, Pietrobon N, Hämmerle CHF, 2002. Visual and spectrophotometric shade analysis of human teeth. J Dent Res. 81(8):578-82.

14. Bahannan Salma, 2014. Shade matching quality among dental students using visual and instrumental methods. J Dent Elsevier. 42(1):48-52.

15. Goel A, VVG P, 2013. Dentofacial analysis in North Indian females. Int J Pure Appl Biosci.;1(3):28-33.

16. Tjan AHL, Miller GD, The JGP, 1984. Some esthetic factors in a smile. J Prosthet Dent. 51(1):24-8.

17. Nold SL, Horvath SD, Stampf S, Blatz MB, 2014. Analysis of select facial and dental esthetic parameters. Int J Periodontics Restorative Dent. 34(5):623-9.

18. Dong JK, Jin TH, Cho HW, Oh SC, 1999. The esthetics of the smile: a review of some recent studies. Int J Prosthodont.12(1):9-19.

19. Moore T, Southard KA, Casko JS, Qian F, Southard TE, 2005. Buccal corridors and smile esthetics. Am J Orthod Dentofac Orthop. 127(2):208-13.

20. Oshagh M, Zarif NH, Bahramnia F, 2010. Evaluation of the effect of buccal corridor size on smile attractiveness. Eur J Esthet Dent. 5:370-80.

21. Silva G, Castilhos E, Masotti A, Junior S, 2012. Dental esthetic self-perception of brazilian dental students. RSBO. 9(4):375-81. 